\title{
Pseudomonas cepacia endocarditis treated with co-trimoxazole and kanamycin
}

\author{
D. C. E. Speller \\ From the Department of Clinical Bacteriology, University Department of Bacteriology, \\ Bristol Royal Infirmary, Bristol
}

Two cases of endocarditis, affecting prosthetic mitral valves, caused by Pseudomonas cepacia, were treated with a combination of co-trimoxazole and kanamycin. Laboratory testing showed that the combination was synergistic and bactericidal to the organism, and its effectiveness was confirmed in treatment of the patients.

An outbreak of infection by Pseudomonas cepacia, previously reported (Speller, Stephens, and Viant, 1971), arising from contaminated aqueous chlorhexidine solutions, included two cases of endocarditis affecting recently-inserted prosthetic mitral valves. The bacterium was resistant to most chemotherapeutic agents, but treatment with a combination of co-trimoxazole (trimethoprim plus sulphamethoxazole) and kanamycin resulted in recovery of one patient and probable eradication of the infection in the other.

\section{Case histories}

\section{Case I}

A 55-year-old man had a mitral valve replacement with a Starr-Edwards prosthesis. Ten days later he began to have intermittent pyrexia, ranging up to $40.6^{\circ} \mathrm{C}$, and the site of an intravenous infusion was observed to be inflamed. $P$. cepacia was isolated from blood culture and in heavy growth from the intravenous cannula. Co-trimoxazole (Septrin tablets, Burroughs Wellcome - $80 \mathrm{mg}$ trimethoprim and $400 \mathrm{mg}$ sulphamethoxazole), 6 daily, were started and his condition improved with return of his temperature to normal.

$\mathrm{He}$ was sent for convalescence to another hospital, and co-trimoxazole was continued for a month. A few days after it was stopped, he again became pyrexial and the same bacterium was isolated from blood culture. Cotrimoxazole was restarted and continued for 6 weeks, but as soon as it was stopped bacteraemia recurred ( 9 separate blood cultures yielding $P$. cepacia) and he was readmitted to this hospital.

$\mathrm{He}$ was given kanamycin $\mathbf{I} \cdot 5 \mathrm{~g}$ daily intravenously, for 7 days, with no response. As a result of the full antibiotic sensitivity testing, described below, co-trimoxazole 8 tablets daily was added, and this was followed by immediate cessation of fever and general improvement. Serum kanamycin concentrations ranged between Io and $20 \mu \mathrm{g}$ per $\mathrm{ml}$; serum dilutions of $\mathrm{I}$ in 4 or $\mathrm{I}$ in 8 Received 22 June 1972. were bactericidal to the pseudomonas; on the 5 th day of combined treatment, a serum specimen taken 3 hours after a dose had a trimethoprim concentration of $\mathbf{3 . 8 2}$ $\mu \mathrm{g}$ per $\mathrm{ml}$, and a sulphamethoxazole concentration of I $40 \mu \mathrm{g}$ per $\mathrm{ml}$. After three weeks of combined treatment the blood urea rose and the kanamycin was discontinued. Three days later kanamycin was not detectable in his serum, the trimethoprim concentration was 4.95 $\mu \mathrm{g}$ per $\mathrm{ml}$, and sulphamethoxazole $200 \mu \mathrm{g}$ per $\mathrm{ml}$.

The patient died a week later of causes unrelated to the infection. There had been no recrudescence of bacteraemia. At necropsy, the valve ring was clear apart from a small vegetation on the ventricular surface. Culture, including enrichment culture in serum broth, of this and a number of specimens from the valve and related tissues failed to yield the bacterium, and no bacteria could be seen in histological sections.

\section{Case 2}

A 48-year-old woman had her incompetent mitral valve replaced by a Starr-Edwards prosthesis, and Io days later began to have an intermittent pyrexia with sweating attacks. The fever began after an infusion of blood, but persisted after the cannula had been removed. Later $P$. cepacia was isolated from three blood cultures taken on consecutive days.

Treatment with co-trimoxazole 8 tablets daily was begun after 8 days of fever. No change in her condition was noted after 3 days. Laboratory testing of the pseudomonas (see below) gave identical results with those of the isolate from Case $I$, and so kanamycin $I \mathbf{g}$ daily was added. Clinical improvement followed at once and her serum became bactericidal to the pseudomonas in the laboratory at a dilution of $I$ in 4. She was discharged from hospital, well, Io weeks after her operation. Cotrimoxazole 6 tablets daily were continued for Io months, and relapse has not occurred in 6 months since the drug was stopped. She suffered no adverse effect from the long course of co-trimoxazole.

Investigation of Pseudomonas cepacia isolates The isolates from both cases behaved identically in 
laboratory tests. Disc antibiotic sensitivity testing indicated resistance to ampicillin, carbenicillin, cephaloridine, chloramphenicol, colistin, gentamicin, streptomycin, tetracycline, sulphonamide alone, and trimethoprim alone, and moderate sensitivity to kanamycin and to co-trimoxazole (the trimethoprim-sulphamethoxazole combination).

The isolates were tested for the bactericidal activity of antibiotics and the effect of combinations by a modification of the method of Garrod and Waterworth (1969). The medium used was nutrient broth, with 5 per cent lysed horse blood, aged overnight at $4^{\circ} \mathrm{C}$. An initial inoculum of $10^{4}$ colony forming units per $\mathrm{ml}$ was treated with the following antibiotics alone and in combinations of two (co-trimoxazole counting as one agent) at the stated concentrations, which were chosen as high, but attainable and acceptable, serum concentrations (given in $\mu \mathrm{g} / \mathrm{ml}$ ). Co-trimoxazole: trimethoprim $5,+$ sulphamethoxazole 100; kanamycin 20; ampicillin 20; carbenicillin 100; colistin 20; gentamicin 15; and streptomycin 20. The effect of the agents was observed by standard loop subculture at 2 hours, 4 hours, and 20 hours. The results were as follows. Ampicillin, carbenicillin, colistin, gentamicin, kanamycin or streptomycin alone: no significant inhibition of growth. Co-trimoxazole alone, or in combination with ampicillin, carbenicillin or colistin: partial bactericidal effect at 20 hours. Co-trimoxazole in combination with kanamycin, gentamicin, or streptomycin: completely killed at 20 hours, with some bactericidal effect at 4 hours (most obvious in the case of the co-trimoxazole-kanamycin combination). All other combinations: no inhibition of growth.

\section{Comment}

The diagnosis of endocarditis depended upon the clinical features and the continued bacteraemia after the removal of the intravenous cannulae which were the source of infection. Case I relapsed after treatment was stopped on two occasions, I month and 3 months after infection had originally occurred. In this outbreak, patients with bacteraemia who did not have prosthetic material in the circulation to be colonized recovered without antibiotic treatment as soon as the cannula, the source of the bacteraemia, was removed.

The results of treatment of bacterial endocarditis parallel laboratory sensitivity test data quite closely providing that the bactericidal effect of chemotherapeutic agents on a comparatively large inoculum of the organism is tested. The usefulness of bactericidal synergistic combinations of agents has been repeatedly shown in endocarditis of natural valves, particularly that caused by faecal streptococci, though the method has often been applied to other organisms (Peard et al., 1970). The investigation reported here illustrates the application of such tests to a very resistant Gram-negative bacillus, causing an infection of a prosthetic valve. The two agents that gave inhibition of the organism on disc testing - co-trimoxazole and kanamycin - did not give satisfactory bactericidal activity alone, but a combination of an aminoglycoside (particularly kanamycin) with co-trimoxazole gave a very synergistic, bactericidal effect. Synergism between cotrimoxazole and kanamycin has been reported as a laboratory finding (Waterworth, 1970) but clinical use of the combination has not been reported.

Co-trimoxazole has been used alone successfully in the treatment of bacterial endocarditis of a natural valve, caused by a sensitive Escherichia coli (Fowle and Zorab, 1970) and in one case of staphylococcal endocarditis affecting a mitral Starr valve (Shafqat, Shah, and Syed, 1971). Another patient in this hospital who had bacteraemia, and probably prosthetic valve endocarditis, caused by a more sensitive pseudomonas strain, recovered after treatment with co-trimoxazole. In the first case described here, though co-trimoxazole suppressed the signs of infection, relapse occurred on two occasions after it was stopped. Kanamycin alone gave little clinical effect, but the combination appears to have eradicated the infection. In Case 2 the long treatment with co-trimoxazole may have been the effective agency in curing the patient, but in the initial stages of her treatment co-trimoxazole gave no clinical effect, while recovery ensued when the combination was given.

I am indebted to Dr. D. W. Barritt and Mr. G. Keen for permission to report on patients under their care; to Dr. S. P. Lapage and Mr. L. R. Hill at the National Collection of Type Cultures for identification of the organisms; and to Dr. A. S. E. Fowle and the Wellcome Research Laboratories for trimethoprim and sulphamethoxazole estimations.

\section{References}

Fowle, A. S. E., and Zorab, P. A. (1970). Esch. coli endocarditis successfully treated with oral trimethoprim and sulphamethoxazole. British Heart fournal, 32, 127.

Garrod, L. P., and Waterworth, P. M. (1969). Tests of combined bactericidal action. Association of Clinical Pathologists, Broadsheet No. 63.

Peard, M. C., Fleck, D. G., Garrod, L. P., and Waterworth, P. M. (1970). Combined rifampicin and erythromycin for bacterial endocarditis. British Medical fournal, 4, 410.

Shafqat, S. H., Shah, S. A. A., and Syed, S. A. (197I). Bacterial endocarditis over prosthetic valves treated with trimethoprim-sulphamethoxazole combination. British Heart fournal, 33, 974 .

Speller, D. C. E., Stephens, M. E., and Viant, A. C. (197I) Hospital infection by Pseudomonas cepacia. Lancet, I, 798 .

Waterworth, P. M. (1970). Sensitivity tests with trimethoprimsulphonamide. South African Medical fournal, 44, Suppl. I5 August, p. 10.

Requests for reprints to Dr. D. C. E. Speller, Department of Bacteriology, Bristol Royal Infirmary, Marlborough Street, Bristol BS2 8HW. 